\title{
Reliably bright mice
}

Mice with inducible reporter genes allow systemic profiling of gene expression throughout the brain.

Researchers led by Hongkui Zeng at the Allen Brain Institute have created and characterized multiple mouse lines in which the Cre-lox system is used to strongly and universally induce fluorescent reporters.

The Cre-lox system is a common genetic tool. In one permutation of this setup, a fluorescent marker gene is placed under the control of a promoter followed by a stop cassette that is 'floxed', or flanked by lox sequences that are recognized by the DNA recombinase Cre. Another part of the setup induces expression of Cre in particular cells; once Cre is induced, both the cells and their progeny express the reporter gene. One option for delivering Cre is to cross mice containing floxed genes with mice in which the recombinase is expressed. The mice are called, respectively, Cre-reporter and Cre-driver lines.
The system is far from perfect. Often Cre is induced unreliably or expression of reporter genes is too weak to detect. Zeng's team sought to generate new Cre-reporter lines and compare their robustness for fluorescence labeling. They placed the reporter genes in the ubiquitously expressed Gt(ROSA)26Sor (Rosa26) locus and included a strong exogenous promoter to drive higher expression of the fluorescent marker gene.

After creating lines expressing several colors, Zeng's team then systematically characterized Cre recombination partners by crossing the reporter lines with 21 Cre-driver lines, including ten that the team specifically created to target cell types in the cerebral cortex. They saw that fluorescence could be tied quite specifically to particular brain regions and brain cell types as well as be used to visualize detailed neuron structure.

The work, which expands the arsenal of Cre-controlled tools for targeting various cell types, is part of a larger goal to find new ways of expressing genetic tools and to produce standardized data that are publicly accessible; such resources are currently lacking for most Cre lines, explains Zeng. "Cell-specific manipulation is getting more and more important in the nervous system," she says, but researchers who create a new system for studying a particular brain region rarely have ready means to conduct or report characterization for the entire brain. Zeng's team already deposited the new Cre-reporter mice with the JAX Mice repository, and characterization data for the mice are available at http://transgenicmouse. alleninstitute.org/. They hope to expand the database with new mouse lines contributed by themselves and by others.

\section{Monya Baker}

\section{RESEARCH PAPERS}

Madisen, L. et al. A robust and high-throughput Cre reporting and characterization system for the whole mouse brain. Nat. Neurosci. 13, 133-140 (2010). 